\title{
Congenital thrombophilia and thrombosis: a study in a single centre
}

\author{
S E Lawson, D Butler, M S Enayat, M D Williams
}

\begin{abstract}
Aim-To identify the incidence of congenital thrombophilia in a cohort of children presenting with symptomatic thromboembolism.

Method-A review of children with thromboembolism investigated for thrombophilia over a 12 month period.

Subjects-Thirty children with thromboembolic episodes and 16 of their family members.

Measurements and data collection-Data were collected on age at diagnosis, underlying diagnosis, site of thrombosis, associated precipitating factors, occurrence of other thromboembolic events, and family history. Investigations included measurement of protein $C$ activity, total and free protein $S$ antigen, antithrombin III activity, screening for factor $V$ Leiden and prothrombin 20210A, urinary homocysteine estimation, and a screen for lupus anticoagulant.
\end{abstract}

Results-Twenty seven of 30 patients had one or more risk factors present at the time of thromboembolism. Eighty three per cent had acquired precipitating factors present, and $43 \%$ had underlying congenital thrombophilia.

Conclusions-There was a high incidence of congenital thrombophilia in this group of patients with symptomatic thromboembolism. These findings emphasise the importance of such defects in the pathogenesis of childhood thrombosis, and suggest that full thrombophilia investigations should be performed on all children presenting with thromboembolic disease.

(Arch Dis Child 1999;81:176-178)

Keywords: thrombosis; congenital thrombophilia

Thromboembolic disease is an infrequent event in childhood, which can be associated with considerable morbidity and mortality. The incidence of venous thromboembolism has been consistently reported at about $0.7 / 100000 /$ year, ${ }^{12}$ with infants and adolescents at greatest risk. In most cases, an underlying precipitating factor is found, and in many, two or more precipitating factors are present. ${ }^{1-4}$

Acquired factors known to predispose to thrombosis are often implicated including central venous lines, malignancy, and sepsis. The role of congenital thrombophilic states is unclear. Several recent studies have reported the incidence of specific inherited prothrombotic conditions in children with thrombosis, although the incidences reported, the populations studied, and the thrombophilic parameters measured have varied considerably. ${ }^{14-7}$

The most common inherited condition known to predispose to thromboembolic disease is the factor $\mathrm{V}$ Leiden mutation (Arg506Gln), which leads to resistance to activated protein C. ${ }^{8}$ Other conditions include deficiencies of protein $\mathrm{C}$, protein $\mathrm{S}$, and antithrombin III, some dysfibrinogenaemias, dysplasminogenaemia, and other fibrinolytic defects. The recently described prothrombin mutation 20210A can also be added to these disorders. ${ }^{9}$ Homocystinuria is also associated with thrombosis in childhood. These different conditions may co-segregate to increase the risk. ${ }^{10-16}$

We have determined the importance of congenital thrombophilic markers and acquired precipitating factors for thrombosis in children who presented to Birmingham Children's Hospital over a 12 month period with thromboembolism, and had investigations for thrombophilia.

\section{Patients and methods}

We reviewed the case notes of all patients who had had a thromboembolic event and had thrombophilia investigations performed at Birmingham Children's Hospital in the 12 month period to December 1997. Data collected included age at diagnosis; underlying diagnosis; site of thrombosis and how it was documented; associated risk factors for thrombosis such as surgery, indwelling central venous line, malignancy, chemotherapy, and sepsis; occurrence of other thrombotic events; and family history. The thrombophilia investigations consisted of measurement of protein C activity, total and free protein $\mathrm{S}$ antigen, antithrombin III activity, screening for factor $\mathrm{V}$ Leiden and prothrombin 20210A, urinary homocysteine estimation, and a screen for lupus anticoagulant.

Protein C was measured using a chromogenic assay (Chromogenix, Möldndal, Sweden) after activation by copperhead snake venom. Protein $S$ was assayed by an enzyme linked immunoabsorbent assay (ELISA), whereby both total and free protein $S$ were measured, the latter after polyethylene glycol precipitation (Dako, Cambridge, UK). Antithrombin III was assayed by a chromogenic method (Chromogenix), after incubation with an excess of factor $\mathrm{Xa}$ and heparin. Age related reference ranges for the above investigations were based on those published by Andrew et $a l .{ }^{17} 18$

Lupus anticoagulant testing consisted of a dilute Russell viper venom time, with confir- 
mation of the presence of a lupus anticoagulant being confirmed by its neutralisation by platelet phospholipid.

Urinary homocysteine was measured by high voltage electrophoresis of amino acids after urine oxidation.

Factor V Leiden mutation detection was carried out using patients' boiled blood in a polymerase chain reaction (PCR), followed by restriction enzyme analysis with $\mathrm{Mnl}$, as described previously. ${ }^{19}$ Detection of the 20210A allele of the prothrombin gene was carried out in the same way, but using the HindIII restriction enzyme. ${ }^{20}$

\section{Results}

Thirty children presenting with symptomatic thromboembolic events had thrombophilia investigations performed during the 12 month study period. Twenty two were investigated after a first thrombotic event and eight after a second or third event. Sixteen family members of seven children also had thrombophilia investigations. Not all family members of each index case were investigated, mainly because of their unavailability.

There were 16 boys and 14 girls. The mean age at diagnosis of the thrombotic event was 6.5 years (range, 1 day to 15 years).

\section{TYPE OF THROMBOSIS}

The thromboses were varied and included cerebrovascular events (11), renal vascular thrombosis (eight), deep vein thrombosis (two), hepatic veno-occlusive disease (two), sagittal sinus thrombosis (two), portal vein thrombosis (one), inferior vena caval thrombosis (one), central venous line associated thrombosis (one), purpura fulminans (one), and pulmonary embolus (one). All were documented by one or more investigations including Doppler ultrasound, computed tomography, magnetic resonance imaging, angiography, histology, or lung ventilation:perfusion scan.

PREDISPOSING FACTORS FOR THROMBOSIS

Twenty seven of 30 patients had one or more predisposing factors at the time of the thrombotic event. Fourteen had an acquired factor alone, two a congenital factor alone, 11 both acquired and congenital factors, and three no identifiable predisposing factors. The most frequently observed acquired factors were a preceding febrile/viral illness or a postoperative state, both seen in nine patients. Four patients had underlying malignant disease, and were receiving chemotherapy through indwelling central venous lines. One child had nephrotic syndrome, one Gram negative sepsis, and one common variable immunodeficiency.

We found an underlying congenital thrombophilic state in 13 patients, although not all patients underwent full thrombophilia investigations. The most common defect was a mutation in the gene encoding factor V Leiden, which was found in four of 30 patients, with all affected children heterozygous. Of those tested, three of 26 were heterozygous for the prothrombin $20210 \mathrm{~A}$ mutation, three of 28 had protein $\mathrm{C}$ deficiency, two of 28 protein $\mathrm{S}$ deficiency, one of 18 homocystinuria, and one of 28 antithrombin III deficiency. None had lupus anticoagulant. One patient was heterozygous for both factor $\mathrm{V}$ Leiden and prothrombin 20210A. The only two patients without additional acquired precipitating factors were one with protein $S$ deficiency, who sustained an iliofemoral deep vein thrombosis (DVT), and one with extrahepatic portal vein thrombosis and protein $\mathrm{C}$ deficiency.

\section{FURTHER THROMBOTIC EVENTS}

Twelve patients suffered more than one thrombotic episode (DVT, three; cerebrovascular event (CVA), three; renal vascular thrombosis (RVT), three; central venous line (CVL) associated thrombosis, one; splenic vein thrombosis, one; retinal artery thrombosis, one). Seven of the further events were the same as the first (DVT, one; CVA, three; RVT, three). The mean time between the first and second thrombotic event was 21 months (range, one month to seven years).

Of those patients experiencing more than one thrombotic event, six had congenital thrombophilia. Of these, two had no other identifiable risk factor at the time of thrombosis (protein C deficiency and prothrombin 20210A). Of the other four, one had homocystinuria and a viral illness, one factor V Leiden and a renal transplant, one antithrombin III deficiency and a viral illness, and one protein $\mathrm{C}$ deficiency and Gram negative sepsis. In the six patients without congenital thrombophilia, acquired precipitating factors were identified in four (viral illness (one), renal transplant (two), common variable immune deficiency and central venous line (one)). Only one of these patients was on anticoagulants (warfarin) at the time of the second event, and the thrombosis was associated with a central venous line and no underlying congenital thrombophilia. Three patients experienced a third event, two of which were associated with an acquired precipitating factor alone (haemodialysis catheter; renal transplant) and one with a congenital thrombophilic state alone (prothrombin 20210A).

\section{FAMILY STUDIES}

Of the 16 family members (seven families) investigated, four had a clinical history of thrombosis, of whom one had congenital thrombophilia (antithrombin III deficiency). Of the 12 without a thrombotic history, eight were found to have an underlying congenital thrombophilic state (factor V Leiden mutation, three; prothrombin 20210A, two; factor V Leiden mutation and protein $\mathrm{S}$ deficiency, one; protein $\mathrm{S}$ deficiency, one; protein $\mathrm{C}$ deficiency, one). In one family without a history of thrombosis, a further thrombophilic defect was found that was not present in the index case.

\section{Discussion}

Thromboembolism is a rare event in childhood, and it's aetiology is usually multifactorial, as is supported by our findings. Precipitating factors in childhood thrombosis are generally acquired and age dependent. In the 
1st year of life, venous thrombosis usually occurs either in association with indwelling venous catheters or as renal vein thrombosis, with the latter being common in the 1 st few days after birth. In older children, central venous lines remain one of the most frequent precipitating factors, and in most studies are the implicated triggering factor in at least $25 \%$ of patients. ${ }^{1-4}$ However, only one patient in our study had a central venous line associated thrombosis.

In the adult population, the incidence of congenital thrombophilic states and the associated risk for thromboembolic events are relatively well established. Inherited thrombophilic states probably also contribute to childhood thrombosis, although their importance has not been determined, and the incidence of inherited prothrombotic states in childhood thrombosis is unclear, with recent reports suggesting incidences of between 3.9\% and $83 \%$. However, in these publications the thrombotic populations studied varied greatly, the number of children studied was often small, not all patients in the study underwent a thrombophilia screen, and the parameters measured varied between the studies. Despite these limitations, congenital thrombophilia does appear to play a role in childhood thrombosis. It tends to occur only when one or more acquired precipitating factor is also present, thereby unmasking the underlying inherited defect. $^{13-7}$

We found a high incidence (43\%) of congenital thrombophilia in this selected group of children presenting with symptomatic thromboembolism. Not all patients were fully investigated so the incidence may be higher than we have reported. We suggest that full thrombophilia investigations should be performed on all children presenting with thromboembolic disease because a considerable number will have an underlying inherited thrombophilic state that may affect their future management. Family studies, although limited, were informative, with a high detection rate of congenital thrombophilia. Family members should be investigated after identification of a child with congenital thrombophilia. This necessitates full counselling of the family with respect to the implications of positive results. The high incidence of multiple thrombotic events in our patients questions the optimal duration of anticoagulation. Half had an underlying congenital thrombophilia, and four of these six had an identifiable acquired risk factor at the time of the second event. Some of these further events might have been prevented by either a longer period of anticoagulation, or selective anticoagulation at times of increased risk of thrombosis. We emphasise the need for controlled clinical trials to determine both the optimal duration and optimal range of anticoagulation in children who have had a thrombotic event.

1 Andrew M, David M, Adams M, et al. Venous thromboembolic complications (VTE) in children: first analyses of the Canadian registry of VTE. Blood 1994; 83:1251-7.

2 Rosendaal FR. Thrombosis in the young: epidemiology and risk factors. A focus on venous thrombosis. Thromb Haemost 1997;78:1-6.

3 David M, Andrew M. Venous thromboembolic complications in children. $\mathcal{F}$ Pediatr 1993;123:337-46.

4 Nuss R, Hays T, Manco-Johnson M. Childhood thrombosis. Paediatrics 1995;96:291-4.

5 Nowak-Gottl U, Dubbers A, Kececioglu D, et al. Factor V Leiden, protein C, and lipoprotein (a) in catheter-related hrombosis in childhood: a prospective study. 7 Paediatr 1997;131:608-12.

6 Sifontes MT, Nuss R, Jacobsen LJ, Griffin JH, MancoJohnson MJ. Thrombosis in otherwise well children with Johnson MJ. Thrombosis in otherwise well children with

7 Sifontes MT, Nuss R, Hunger SP, Wilimas J, Jacobson LJ, Manco-Johnson MJ. The factor V Leiden mutation in children with cancer and thrombosis. Br F Haematol 1997;96: $484-9$.

8 Dahlback B, Carlsson M, Svensson PJ. Familial thrombophilia due to a previously unrecognised mechanism characterised by poor anticoagulant response to activated protein C: prediction of a cofactor to activated protein C. Proc Natl Acad Sci USA 1993;90:1004.

9 Poort SR, Rosendaal FR, Reitsma PH, Berina RM. A common genetic variation in the 3'-untranslated region of the prothrombin gene is associated with elevated plasma prothrombin levels and an increase in venous thrombosis. Blood 1996;88:3698-703.

10 Ehrenforth S, Ludwig G, Klinke S, Krause M, Scharrer I, Nowak-Gottl U. The prothrombin 20210A allele is frequently coinherited in young carriers of the factor V Arg 506 to Gln mutation with venous thrombophilia. Blood 1998;91:2209-10.

11 Gandrille S, Greengard JS, Alhenc-Gelas M, et al. Incidence of activated protein $\mathrm{C}$ resistance caused by the ARG 506
GLN mutation in factor $\mathrm{V}$ in 113 unrelated symptomatic protein C deficient patients. Blood 1995;86:219-24.

12 Koeleman BPC, Reitsma PH, Allaart CF, Bertina RM. Activated protein $\mathrm{C}$ resistance as an additional risk factor for thrombosis in protein C deficient families. Blood 1994; 84:1031-5.

13 Seligsohn U, Zivelin A. Thrombophilia as a multigenic disorder. Thromb Haemost 1997;78:297-301.

14 van Boven HH, Reitsma PH, Rosendaal FR, et al. Factor V Leiden (FV R506Q) in families with inherited antithrombin deficiency. Thromb Haemost 1996;75:417-21.

15 Zoller B, Berntsdotter A, de Frutos PG, Dahlback B. Resistance to activated protein $\mathrm{C}$ as an additional genetic risk factor in hereditary deficiency of protein S. Blood 1995;85: 3518-23.

16 Zoller B, Svensson PJ, Dahlback B, Hillarp A. The A20210 allele of the prothrombin gene is frequently associated with the factor V Arg 506 to Gln mutation but not with protein $S$ deficiency in thrombophilic families. Blood 1998;91: S210-11.

17 Andrew M, Vegh P, Johnston M, Bowker J, Ofosu F, Mitchell L. Maturation of the haemostatic system during childhood. Blood 1992;80:1998-2005.

18 Andrew M. Developmental haemostasis: relevance to newborns and infants. In: Nathan DG, Orkin SH, eds. Nathan and Oski's haematology of infancy and childhood, Vol. 1, 5th ed. Philadelphia: WB Saunders, 1998:119-20.

19 Enayat MS, Williams MD, Hill FGH. Further simplification of the factor V:Q506 mutation detection test. Blood Coagul Fibrinolysis 1997;8:205.

20 Makris M, Preston FE, Beauchamp NJ, et al. Co-inheritance of the 20210A allele of the prothrombin gene increases the risk of thrombosis in subjects with familial thrombophilia. Thromb Haemost 1997;78:1426-9. 